\title{
La lipochimie et le carbone renouvelable : une opportunité pour l'agro-industrie
}

\author{
Antoine GASET \\ Laboratoire de chimie agro-industrielle, \\ UMR 1010 Inra/INPT - Ensiacet, \\ 118 , route de Narbonne - 31077 Toulouse \\ cedex 4 \\ $<$ Antoine.Gaset@ensiacet.fr>
}

\begin{abstract}
The willing of the Member States of the European Union to reduce the petrol dependence, associated to the environment quality problems and the search for better life conditions for citizens sets the lipochemistry within the expectations of the sustainable development and within the hopes of our society. The agriculture will thus play a capital role in the coming years. Indeed, the oleaginous will have a more and more privileged position to reply of course to the energy requirements but also mainly to supply specialty and commodity bio-products.

In these conditions, the industry of the renewable carbon, and particularly the industry of oleaginous, are going to develop and will come across an expansion in the short and the medium term.

In this new context, the synergy between different disciplines will be a provider of success. Also, the "assembling" of different agricultural productions will be a source of innovation and a basis for the creation of new economic activities. In this sense, the reactivity between the lignocellulosic substrates and the fatty derivatives constitutes one of these challenges. We have investigated new activation techniques adapted to two-phase solid/liquid media. These activations employ either technological ways that favour the compatibility between products and substrates, or fatty derivatives that provide a good reactivity after appropriate functionalisation. The examples that are given try to illustrate this approach, to debate around new concepts, and to place emphasis on all the scientific and economic benefits obtained therefrom. From then on, the lipochemistry is situated in the centre of a system where the deductive research and the inductive research nourish each other.
\end{abstract}

Key words: fatty acids, fatty esters, fatty derivatives, cellulose, lignocellulose, two-phase solid/liquid medium

solvants susceptibles d'avoir un impact négatif soit sur la santé soit sur l'environnement.

Si cette chimie verte fait, en plus, appel aux matières premières renouvelables et plus particulièrement aux oléagineux, elle présente tous les critères qui permettent de parler de lipochimie verte et de placer celle-ci dans l'ère du carbone renouvelable et dans l'ère de l'écoconception qui déjà caractérisent le $\mathrm{XXI}{ }^{\mathrm{e}}$ siècle. Dans ces conditions, cette chimie verte des oléagineux se caractérise donc par ses aspects sociétal, environnemental et économique. Elle nous permet de parler de la lipochimie durable. Une telle démarche est maintenant renforcée et crédibilisée par la réglementation Reach et également par la prise en compte de la négociation des quotas de $\mathrm{CO}_{2}$.

Tous ces éléments confortent la politique de recherche et d'innovation qui renforce la place de la lipochimie dans notre économie. En effet, elle devrait permettre d'avoir un impact positif sur les futures orientations de l'exploitation des terres agricoles conjointement à la prise en compte de la réforme de la PAC. C'est, semblet-il, une opportunité que le monde agricole et le monde industriel vont saisir pour répondre aux attentes économiques et environnementales de notre société.

La lipochimie, vecteur de richesses, c'est le défi que nous devons relever.

Dans ce contexte, nous allons focaliser notre attention sur un verrou scientifique et sur les premières solutions qui lui sont proposées.

Comment faire réagir de façon efficace deux entités qui n'ont aucune affinité : les lignocelluloses et les huiles végétales? Deux substrats que tout oppose, I'un est solide et hydrophile, I'autre est liquide et hydrophobe. Comment faire cohabiter ces deux partenaires dans des conditions de bonne réactivité?

Quels sont les moyens technologiques que I'on peut explorer ? Quels sont les dérivés lipidiques dont les fonctions permettent d'atteindre cet objectif?

Les informations présentées sont issues d'une partie des travaux menés dans ce secteur par le laboratoire de chimie agro-industrielle UMR 1010 Inra/INP-Ensiacet. Elles constituent une participation très modeste à la réponse qu'attend cette très importante question.

Lorsque l'on envisage de faire réagir les groupements hydroxyles de la cellulose sur un 
dérivé lipochimique, on se heurte immédiatement à l'existence d'un milieu réactionnel biphasique solide-liquide. Les caractéristiques physiques et chimiques opposent les deux substrats et rendent la réaction inopérante. Comment réduire ces différences? Comment pouvons-nous les rendre compatibles l'une par rapport à l'autre ? Quelles techniques ou quelles structures chimiques pouvons-nous mettre en œuvre afin que substrat et réactif se tolèrent et puissent ainsi réagir l'un sur l'autre?

Dès que ce verrou scientifique sera levé il deviendra alors possible d'atteindre l'objectif visé et $d^{\prime}$ avoir accès à des applications originales.

Pour tenter d'apporter des réponses à ces interrogations nous avons choisi de nous appuyer sur quelques exemples qui, en outre, nous permettront d'illustrer la démarche suivie.

Nous avons été amenés à rechercher de nouveaux modes d'activations qui soient adaptés à ces milieux polyphasiques solide-liquide. Ces modes d'activations ont recours soit à des moyens technologiques qui favorisent la compatibilité des substrats, soit à des dérivés lipochimiques qui offrent une bonne réactivité grâce à une judicieuse fonctionnalisation de ceux-ci.

\section{Amélioration de la réactivité de molécules lipidiques par des modes d'activations adaptés aux milieux réactionnels biphasiques}

Les composés lipidiques, dérivés d'huiles végétales (acides gras, esters d'acides gras, triglycérides, monoglycérides, etc.) sont, a priori, capables de réagir avec les fonctions hydroxyles de la cellulose ou de la matière lignocellulosique par des réactions d'acylation.

Cell- $\mathrm{OH}+\mathrm{CH}_{3}-\left(\mathrm{CH}_{2}\right)_{n}-\underset{\mathrm{O}}{\mathrm{C}}-\mathrm{O}-\mathrm{R} \rightleftarrows \mathrm{Cell}-\mathrm{O}-\mathrm{C}-\left(\mathrm{CH}_{2}\right)_{n}-\mathrm{CH}_{3}+\mathrm{R}-\mathrm{OH}$

Toutefois, le fait de faire réagir le réactif lipidique, peu polaire et hydrophobe avec un substrat cellulosique très polaire et hydrophile, constitue incontestablement un verrou technologique important. Pour apporter une solution à ce dernier plusieurs stratégies ont été adoptées.

Cell $-\mathrm{OH}+\mathrm{Cl}-\underset{\|}{\mathrm{C}}-\left(\mathrm{CH}_{2}\right)_{n}-\mathrm{CH}_{3} \underset{\text { pyridine }}{\stackrel{-\mathrm{HCl}}{\rightleftarrows}}$ Cell $-\mathrm{O}-\underset{\|}{\stackrel{C}{-}-\left(\mathrm{CH}_{2}\right)_{n}-\mathrm{CH}_{3}}$

L'utilisation de chlorure d'acides gras permet d'atteindre l'objectif attendu. En effet des degrés de substitution proches de 3 sont atteints après optimisation des conditions opératoires [1].

On a montré que, grâce à ces réactions, les fibres lignocellulosiques deviennent hydrophobes, thermoplastiques et thermiquement plus stables. Une telle réaction permet d'avoir accès à de nouveaux matériaux polyfonctionnels à matrice cellulosique dont les propriétés ont été évaluées [2, 3].

Toutefois, le dégagement d'acide chlorhydrique et la présence de pyridine ne sont pas compatibles avec les canons de la chimie verte. De plus, il faut noter que l'extrapolation de cette réaction à l'échelle industrielle va intensifier ce reproche. Par ailleurs, I'acide chlorhydrique formé dégrade une partie des fibres végétales même si un courant d'azote est utilisé pendant la réaction pour réduire le temps de contact de l'acide. D'autres voies d'accès ont donc été recherchées. Nous avons choisi de rapporter quatre d'entre elles; l'échange de solvants, le réacteur thermo-mécano-chimique bi-vis, les microémulsions réactives, les microémulsions réactives couplées à une activation micro-ondes qui, à des degrés différents, permettent d'apporter des éléments de réponse.

\section{L'échange de solvants}

L'échange de solvants est un procédé connu pour sécher la nitrocellulose et la cellulose sans observer les phénomènes de cornification de celle-ci. On sait que l'utilisation de méthanol et d'hexane permet de conserver les propriétés initiales de la cellulose. Cette information a été extrapolée dans le but de favoriser la réaction d'acylation de la cellulose par des esters méthyliques d'acides gras ou par des acides gras. Dans un premier temps, on procède à une imprégnation de la cellulose par de l'eau puis, dans un deuxième temps, celle-ci est déplacée par extraction solide-liquide avec de l'éthanol. Enfin, le réactif lipidique (acide gras, ester d'acide gras, triglycéride) permet de déplacer l'éthanol par extraction solide-liquide. Dans ces conditions, le nouveau milieu réactionnel n'est alors constitué que par le dérivé lipidique, par la fibre cellulosique et par le catalyseur. Ce dernier est choisi acide ou basique selon le dérivé lipidique concerné. L'eau et l'éthanol utilisés conservent eux aussi le caractère chimie verte à ce "procédé compatibilisant ». Ainsi, grâce à ces échanges successifs, le réactif lipidique et le réseau de la cellulose sont mis en contact. La réaction menée avec un ester d'acide gras en présence d'un catalyseur basique conduit à un degré de substitution de 0,2. Bien qu'en apparence faible, cette valeur s'est avérée suffisante pour conférer aux fibres cellulosiques le caractère hydrophobe mais pas assez élevée pour donner un caractère thermoplastique au nouveau agromatériau $[4,5]$.

II est important de souligner que pour la première fois la réaction de transestérification entre les triacylglycérol et la cellulose a été réalisée sans ajout de solvant en milieu hétérogène solide-liquide. Or, ce résultat est grevé par les volumes de solvants qu'il est nécessaire de mettre en œuvre (cellulose : 1, solvant : 50) et par le coût lié à leur recyclage dans l'optique d'une application industrielle. C'est pour cela qu'il nous est apparu que le réacteur bi-vis pourrait être une alternative au prétraitement d'échange de solvants en favorisant le contact intime entre les phases solide et liquide. On peut également penser que les effets mécaniques subis par la matière végétale réduiront le nombre de liaisons hydrogènes dans la phase cristalline de la cellulose. La réactivité entre le triglycéride et les fonctions hydroxyles devrait être facilitée.

\section{Le réacteur thermo-mécano-chimique bi-vis}

La réaction de transestérification entre les triglycérides issus des huiles végétales et la cellulose en l'absence de solvant a pu être envisagée pour la première fois grâce aux hautes températures et aux valeurs des pressions microlocalisées qui sont obtenues avec un réacteur extrudeur bi-vis dont le profil des vis a été étudié et adapté. Les contraintes thermiques, mécaniques et chimiques de ce " procédé compatibilisant » permettent de favoriser un contact intime entre le substrat hydrophile et le réactif hydrophobe et d'améliorer ainsi leur réactivité dans ce milieu hétérogène solide-liquide. La réaction de transestérification de l'huile de palmiste par de la sciure de pin micronisée (inférieure à $10 \mu \mathrm{m}$ ) a été menée dans des conditions originales (température à l'entrée du réacteur: $135^{\circ} \mathrm{C}$, température dans le réacteur : $180^{\circ} \mathrm{C}$ à $200{ }^{\circ} \mathrm{C}$, temps de séjour : 90 secondes). Elle conduit à des taux d'ester de 1300 meq par kilo de sciure et à un caractère hydrophobe de celle-ci (angle de contact statique : $95^{\circ}$ ).

La comparaison des résultats obtenus dans des conditions identiques en mettant en œuvre soit un réacteur classique soit le réacteur thermomécano-chimique, font nettement apparaître l'efficacité et les avantages de ce dernier (tableau 1).

Cette technologie qui relève parfaitement de la chimie verte du carbone renouvelable permet de proposer une méthode de transestérification entre une poudre lignocellulosique et une huile végétale sans ajout de solvant. De plus, elle présente l'avantage de mettre en œuvre un catalyseur basique également d'origine végétale (laurate de sodium) et aussi de pouvoir réaliser en régime continu cette réaction hétérogène solide-liquide.

L'utilisation du laurate de sodium a été retenue par la suite pour étudier un « procédé de compatibilisation » mettant en jeu une microémulsion réactive. 
Tableau 1. Réactions de transestérification entre la cellulose et l'huile de palmiste.

\begin{tabular}{|c|c|c|c|c|c|}
\hline \multicolumn{3}{|c|}{ Réacteur classique } & \multicolumn{3}{|c|}{ Réacteur thermo-mécano-chimique } \\
\hline Conditions de réaction & Taux d'ester (meq/kg) & Angle de contact & Conditions de réaction & Taux d'ester (meq/kg) & Angle de contact \\
\hline $180^{\circ} \mathrm{C} / 6 \mathrm{~h}$ & 0 & $0^{\circ}$ & $200{ }^{\circ} \mathrm{C} / 45 \mathrm{sec}$ & 500 & $10^{\circ}$ \\
\hline $190^{\circ} \mathrm{C} / 6 \mathrm{~h}$ & 0 & $0^{\circ}$ & $200^{\circ} \mathrm{C} / 90 \mathrm{sec}$ & 1300 & $95^{\circ}$ \\
\hline $200{ }^{\circ} \mathrm{C} / 6 \mathrm{~h}$ & Dégrad. & $0^{\circ}$ & $200^{\circ} \mathrm{C} / 180 \mathrm{sec}$ & Dégrad. & $0^{\circ}$ \\
\hline
\end{tabular}

\section{Les microémulsions réactives}

La mise au point de microémulsions formées avec le réactif lipidique (acide gras ou ester $\mathrm{d}^{\prime}$ acide gras) un sel acide gras et l'eau nous a permis de mettre en œuvre un procédé original d'hydrophobation des fibres végétales. Cette émulsion permet en effet d'améliorer la pénétration du réactif et du catalyseur à l'intérieur de la phase solide constituée par les fibres cellulosiques et de créer ainsi un micromilieu réactionnel après élimination de l'eau à $130^{\circ} \mathrm{C}$, propice soit à la réaction d'estérification soit à la réaction de transestérification selon que le réactif lipidique choisi est l'acide gras ou l'ester d'acide gras.

Le processus réactionnel a été étudié à partir de la réaction modèle : acide octonoïque/laurate de potassium puis généralisé à d'autres acides gras (figure 1).

Après optimisation de la réaction [6] un degré de substitution satisfaisant de 0,3 est obtenu qui confère à la fibre cellulosique un bon caractère hydrophobe $\left[\theta\left(^{\circ}\right): 85^{\circ}\right]$.

La comparaison de plusieurs sels d'acide gras en tant que catalyseur a fait ressortir que la nature du cation semble modifier les valeurs du DS. Les sels potassiques s'avèrent être un peu plus efficaces que les sels sodiques (tableau 2).

II faut noter que les études menées sur les échantillons de fibres ou de tissus de coton traités par les acides gras font ressortir un caractère hydrophobe marqué (tableau 2), et ceci quelle que soit la longueur de la chaîne aliphatique greffée (tableaux 3 et 4).

Il a été démontré que :

- grâce aux systèmes créés par les microémulsions les réactions d'estérification et de transestérification ont pu être réalisées dans un milieu hétérogène solide-liquide.

* L'estérification de la cellulose par l'acide octanoïque $\left(10 \mathrm{eq} / \mathrm{eqOH}\right.$ cell $\left.-195^{\circ} \mathrm{C}\right)$ associé à des quantités de laurate de potassium et $\mathrm{d}^{\prime}$ eau respectivement de $1.10^{-2}$ et $3,6 \mathrm{eq} / \mathrm{eq}$ $\mathrm{OH}$ cell conduit à un octanoate de cellulose ayant un DS de 0,28 en 6 heures.

* La transestérification de la cellulose par le laurate de méthyle $(7,6 \mathrm{eq} / \mathrm{eq} \mathrm{OH}$ cell) en présence de laurate de sodium $(2,1.10-2)$ et d'eau (1,2 eq/eq OH cell) conduit à un DS de 0,13 après 6 heures de réaction à $195^{\circ} \mathrm{C}$.

- le sel d'acide gras joue un double rôle : celui de catalyseur et celui de tensioactif.

- le catalyseur est autorégénéré dans la réaction et que le catalyseur initial joue aussi le rôle de réactif. En effet, en fin de réaction, nous avons identifié des chaînes en C8 greffées sur la cellulose à côté de celles en $\mathrm{C} 12$ normalement attendues.

- le rôle du taux d'eau présent dans le système réactionnel est déterminant sur l'avancement de la réaction. Un taux d'eau trop faible n'est par favorable. Un taux d'eau trop élevé est

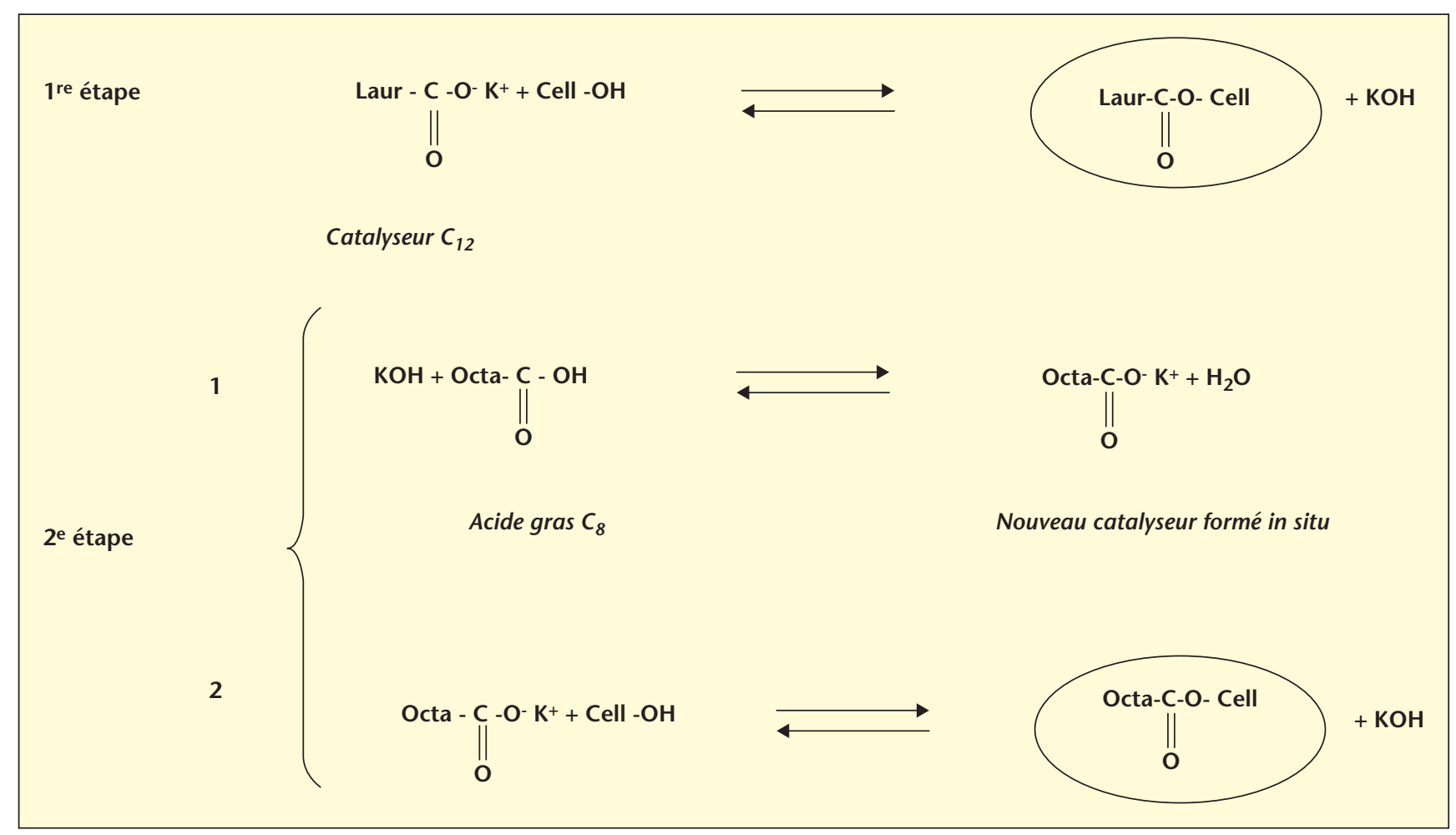

Figure 1. Hydrophobation des fibres végétales : processus réactionnel. 
Tableau 2. Influence de la nature de sel d'acide gras sur l'estérification de la cellulose par l'acide octanoïque.

\begin{tabular}{|ll|}
\hline Sels d'acide gras & DS \\
\hline Aucun & 0 \\
Laurate de sodium & 0,23 \\
Laurate de potassium & 0,28 \\
Stéarate de sodium & 0,20 \\
Stéarate de potassium & 0,26 \\
\hline
\end{tabular}

Acide octanoïque : 10 eq/eq OH cell, sel d'acide gras 0,01/eq/eq OH cell, eau 3,6 eq/eq OH cell ; $\mathrm{T}^{\circ}: 195^{\circ} \mathrm{C}$; $t=6$ heures.

Tableau 3. Généralisation de la réaction d'estérification de la cellule par l'acide octanoïque aux fibres de coton catalysée par le laurate de potassium (conditions opératoires identiques à celles indiquées dans le tableau 2).

\begin{tabular}{|lcc|}
\hline Substrats & DS & $\boldsymbol{\theta}$ ( $\left.^{\circ}\right)$ \\
\hline$\alpha$-cellulose & 0,28 & 80 \\
Fibres de coton & 0,21 & 85 \\
Tissus de coton & 0,18 & 110 \\
\hline
\end{tabular}

Tableau 4. Angles de contact des tissus traités par les réactions d'acylation (DIGIROP équipé d'un analyseur d'images) (conditions opératoires du tableau 2).

\begin{tabular}{|lcc|}
\hline Prétraitement & Réactif & $\left.\boldsymbol{\theta} \mathbf{(}^{\circ}\right)$ \\
\hline Aucun (blanc) & Aucun & 0 \\
Émulsion & Acide C8 & $115-120$ \\
Émulsion & Acide C18 & $105-110$ \\
\hline
\end{tabular}

défavorable car il favorise alors la réaction inverse de saponification.

- les sels d'acide gras évitent la dégradation du polymère cellulosique.

- la réaction pouvait être généralisée aux acides gras traditionnels (caprique, myristique, palmitique, stéarique, oléique...).

- la possibilité de recycler plusieurs fois le milieu réactionnel.

- la réaction pouvait être généralisée à différents substrats cellulosiques.

\section{Les microémulsions réactives couplées à une activation micro-ondes}

Dans un deuxième temps, la technique des microémulsions a été exploitée en mettant en œuvre un réacteur dont le chauffage était assisté par micro-ondes. Nous avons pu com- parer les nouveaux résultats à ceux que nous avions obtenu avec un chauffage classique (tableaux 5 et 6).

L'utilisation de ce type de chauffage nous a permis :

- de réduire considérablement la durée de la réaction (de 6 heures à 5 minutes) ;

- d'atteindre des valeurs des DS comparables à ceux issus d'essais réalisés avec un chauffage classique;

- d'observer (MEB) une faible dégradation du substrat cellulosique ;

- de recycler plusieurs fois (5) avec succès la phase liquide du milieu réactionnel ;

- de réaliser l'acylation de protéines [7] ;

- d'appliquer aisément cette réaction d'acylation aux fibres de cellulose, aux fils et aux tissus de coton dans des conditions tout à fait propi- ces à un développement à plus grande échelle de ce " procédé propre » de greffage de châ̂nes grasses,

- de valider la faisabilité de ce procédé d'acylation dans un réacteur pilote micro-ondes fonctionnant en continu [8].

Sur un plan pratique, ces fibres cellulosiques ou ces tissus de coton hydrophobés permettent $d^{\prime}$ envisager diverses applications. On peut citer à titre d'exemples : le secteur de l'habillement, le secteur de la construction ou le domaine de la filtration, etc.

Au-delà de la mise en œuvre de « technologies compatibilisantes", nous avons aussi tenté d'améliorer la réactivité de la cellulose grâce à I'utilisation de structures lipidiques plus réactives, telles que par exemple les anhydrides mixtes et les anhydrides d'alkényles succiniques.

\section{Exploitation de dérivés lipidiques compatibles avec des milieux réactionnels biphasiques solide-liquide}

\section{Les anhydrides mixtes lipidiques}

Nous savons que les acides gras ne réagissent pas directement sinon très mal sur la cellulose selon des processus de catalyse acide. Afin de contourner cette difficulté, l'anhydride acétique a été utilisé comme co-réactif pour former in situ un réactif intermédiaire doté d'une bonne réactivité : un anhydride mixte « acétique-lipidique». L'augmentation du caractère polaire de cette molécule a permis d'améliorer sa compaptibilité avec les groupements hydroxyles de la cellulose et donc sa réactivité (figure 2).

À partir de cette nouvelle méthode appliquée en milieu hétérogène solide-liquide (tableau 7)

[5] et comparée aux résultats obtenus en milieu homogène (tableau 8) [4] nous avons obtenu un nouveau type de biopolymère peu exploré dans la littérature: les esters mixtes « acétiques-lipidiques » de cellulose.

Ces nouveaux agromatériaux possèdent non seulement un caractère hydrophobe remarquable mais également des propriétés thermo-

Tableau 5. Réactions d'acylation entre la cellulose et des réactifs lipidiques.

\begin{tabular}{|c|c|c|c|c|c|c|c|}
\hline \multicolumn{4}{|c|}{ Réacteur classique } & \multicolumn{4}{|c|}{ Réacteur à chauffage assisté par micro-ondes } \\
\hline Réactif & Conditions de réaction & DS & $\begin{array}{l}\text { Angle de } \\
\text { contact }\end{array}$ & Réactif & Conditions de réaction & DS & $\begin{array}{l}\text { Angle de } \\
\text { contact }\end{array}$ \\
\hline Acide & $195^{\circ} \mathrm{C} / 6$ h sans catalyseur & 0 & $0^{\circ}$ & Acide octanoique & $195^{\circ} \mathrm{C} / \mathbf{5}$ min sans catalyseur & 0 & $10^{\circ}$ \\
\hline Octanoique & $\begin{array}{l}195^{\circ} \mathrm{C} / \mathbf{6} \mathbf{~ h} \text { avec laurate } \\
\text { de potassium }\end{array}$ & 0,28 & $80^{\circ}$ & & $\begin{array}{l}195^{\circ} \mathrm{C} / \mathbf{5} \mathbf{~ m i n} \\
\text { avec laurate de potassium }\end{array}$ & 0,28 & $80^{\circ}$ \\
\hline \multirow[t]{2}{*}{$\begin{array}{l}\text { Octanoate de } \\
\text { méthyle }\end{array}$} & $195^{\circ} \mathrm{C} / 6$ h sans catalyseur & 0 & $0^{\circ}$ & $\begin{array}{l}\text { Octanoate de } \\
\text { méthyle }\end{array}$ & $\begin{array}{l}195^{\circ} \mathrm{C} / \mathbf{5} \text { min } \\
\text { sans catalyseur }\end{array}$ & 0 & $0^{\circ}$ \\
\hline & $\begin{array}{l}195^{\circ} \mathrm{C} / 6 \mathrm{~h} \text { avec laurate } \\
\text { de potassium }\end{array}$ & 0,12 & $90^{\circ}$ & & $\begin{array}{l}195^{\circ} \mathrm{C} / \mathbf{5} \mathbf{~ m i n} \\
\text { avec laurate de potassium }\end{array}$ & 0,11 & $90^{\circ}$ \\
\hline
\end{tabular}


Tableau 6. Effet de l'acide gras sur I'acylation de la cellulose avec l'anhydride mixte. En milieu hétérogène solide-liquide.

\begin{tabular}{|lcccc|}
\hline Acide gras & $\mathbf{n}$ & DSn (lipidique) & DS2 (acétique) & DS total $^{*}$ \\
\hline Caprylique & 8 & 0,30 & 0,76 & 1,06 \\
Caprique & 10 & 0,28 & 0,75 & 1,03 \\
Laurique & 12 & 0,25 & 0,68 & 0,93 \\
Myristique & 14 & 0,22 & 0,64 & 0,86 \\
Palmitique & 16 & 0,19 & 0,55 & 0,74 \\
Stearique & 18 & 0,10 & 0,31 & 0,41 \\
\hline
\end{tabular}

* Des DS totaux d'une valeur de 3 peuvent être obtenus en mettant en œuvre une température plus élevée.

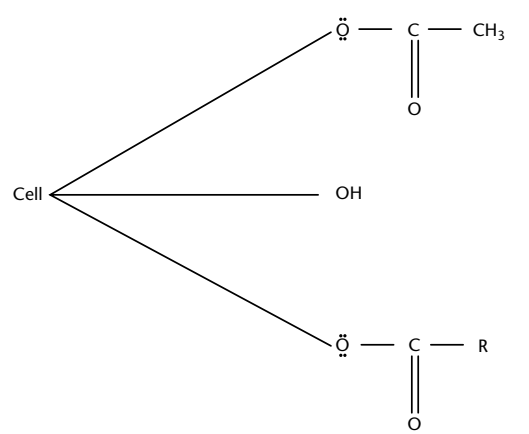

plastiques qui sont directement liées aux valeurs des degrés de substitution (DS) obtenues. Après optimisation ils peuvent atteindre des valeurs proches de la valeur maximale de 3 . L'acquisition de toutes ces connaissances scientifiques a permis de rendre possible la réactivité entre deux partenaires antagonistes (les huiles végétales et la cellulose) et de déboucher sur un nouvel agromatériau totalement issu du monde végétal. II s'agit-là d'un bioproduit obtenu par un procédé vert dont les retombées technico-économiques sont importantes surtout au niveau du traitement des bois. Elles peuvent être résumées en retenant les apports principaux.

Les différentes essences de bois traités par les anhydrides « mixtes-lipidiques » acquièrent : - une remarquable stabilité dimensionnelle à I'eau, aux UV et aux variations de températures, on parle alors d'un support stable ;

- une conservation totale de toutes les propriétés mécaniques du bois ;

- une fonctionnalisation du matériau lignocellulosique compatible avec les opérations de collage ;

- une finition qui sera garantie 10 ans ;

- une excellente aptitude au vieillissement. Placés en situation de simulation de vieillissement accéléré, ces bois conservent intactes leurs propriétés après 30 ans.

Ces travaux ont ouvert une nouvelle voie d'exploitation des oléagineux qui a le mérite d'être sans impact à la fois sur l'environnement et sur la santé de l'homme [9].

Nous voyons que la lipochimie traitée de façon transversale, c'est-à-dire en synergie avec d'autres constituants de la matière végétale, ouvre de nouveaux concepts scientifiques et peut ouvrir de nouvelles portes à notre économie en favorisant une enrichissante contribution du monde agricole, tant au niveau des types d'huiles végétales les mieux adaptés qu'à celui de leurs implications dans les opérations à plus fortes valeurs ajoutées.

\section{Les anhydrides d'alkényles succiniques lipidiques : les oléo-ASA}

Les anhydrides d'alkényles succiniques (ASA) sont des molécules de synthèse issues de la ène-réaction entre l'anhydride maléique (énophile) et un alcène non conjugué possédant un hydrogène en position allylique (R1 et R2 représentant des atomes d'hydrogène ou bien des chaînes aliphatiques) (figures 3 et 4).

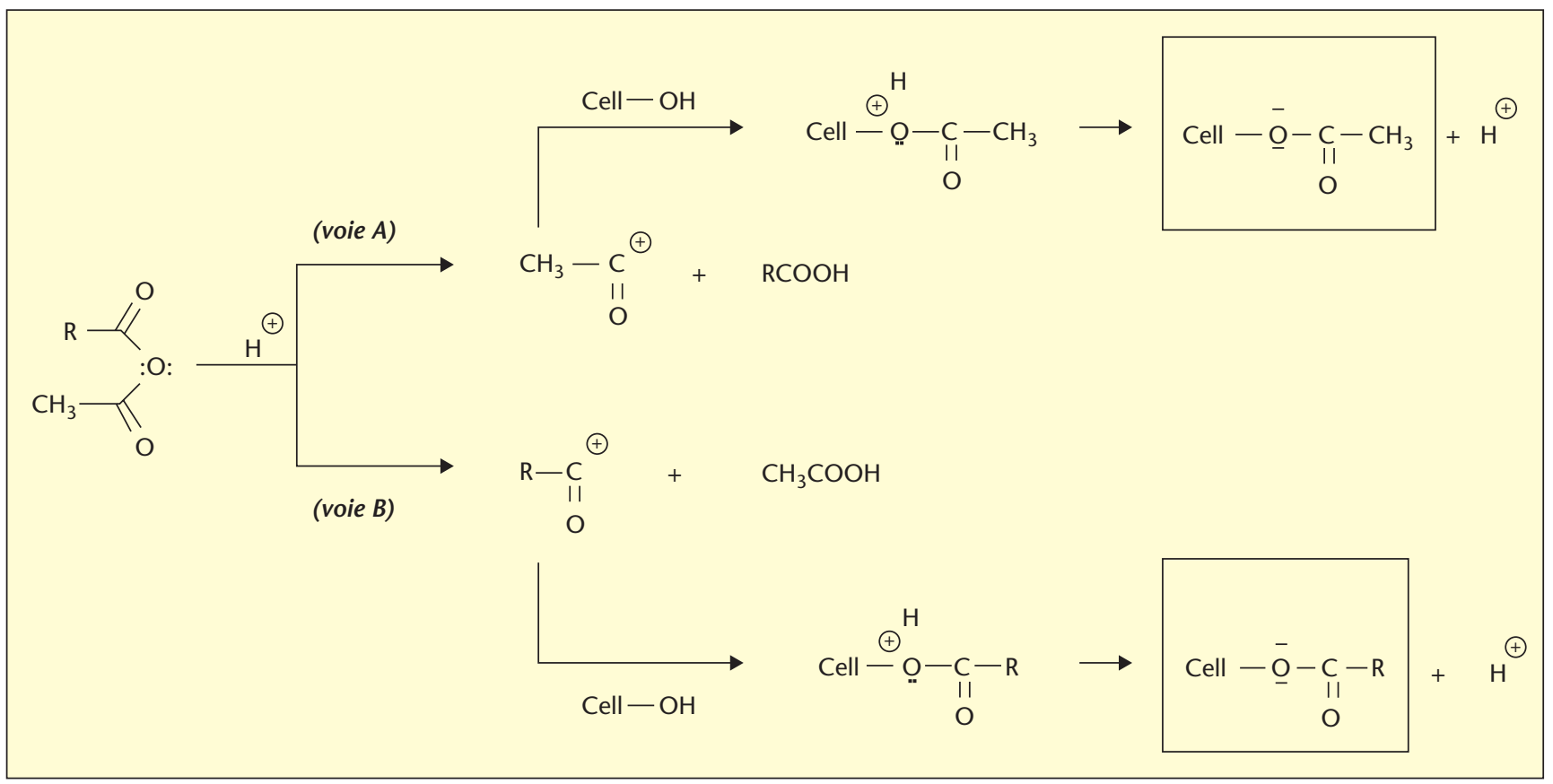

Figure 2. Anhydride mixte acétique-lipidique. 
Tableau 7. Effet de la nature de l'acide gras sur le mélange d'acylation de la cellulose avec l'anhydride mixte en milieu hétérogène solide-liquide $\left(T^{\circ}=110^{\circ} \mathrm{C}, t=2 \mathrm{~h}, \mathrm{C}[\right.$ lauréate $\mathrm{de} \mathrm{k}+\mathrm{]}=7 \mathrm{meq} / \mathrm{OH})$.

\begin{tabular}{|lcccc|}
\hline Acide gras & $\mathbf{n}$ & DSn (lipidique) & DS2 (acétique) \\
\hline Caprylique & 8 & 0,30 & 0,76 & 0,75 \\
Caprique & 10 & 0,28 & 0,68 & 1,06 \\
Laurique & 12 & 0,25 & 0,64 \\
Myristique & 14 & 0,22 & 0,93 \\
Palmitique & 16 & 0,19 & 0,55 \\
Stearique & 18 & 0,10 & 0,31 \\
\hline
\end{tabular}

* Des DS totaux d'une valeur de 3 peuvent être obtenus en mettant en œuvre une température plus élevée.

Tableau 8. Synthèse de triesters de cellulose (acétate-alkanoate) à partir d'acides gras et d'acide acétique en milieu homogène (4Licl/N,N-diméthylacétamide).

\begin{tabular}{|lccc|}
\hline Substituant lipidique & DSa acétique & DSn lipidique & DS total \\
\hline n-octanoïque & 2,19 & 0,81 & 3,00 \\
n-decanoïque & 2,18 & 0,82 & 3,00 \\
n-dodecanoïque & 2,18 & 0,82 & 3,00 \\
n-tetradecanoïque & 2,27 & 0,72 & 2,70 \\
n-hexadecanoïque & 2,24 & 0,74 & 2,66 \\
n-octadecanoïque & 2,24 & 0,71 & 3,15 \\
\hline
\end{tabular}

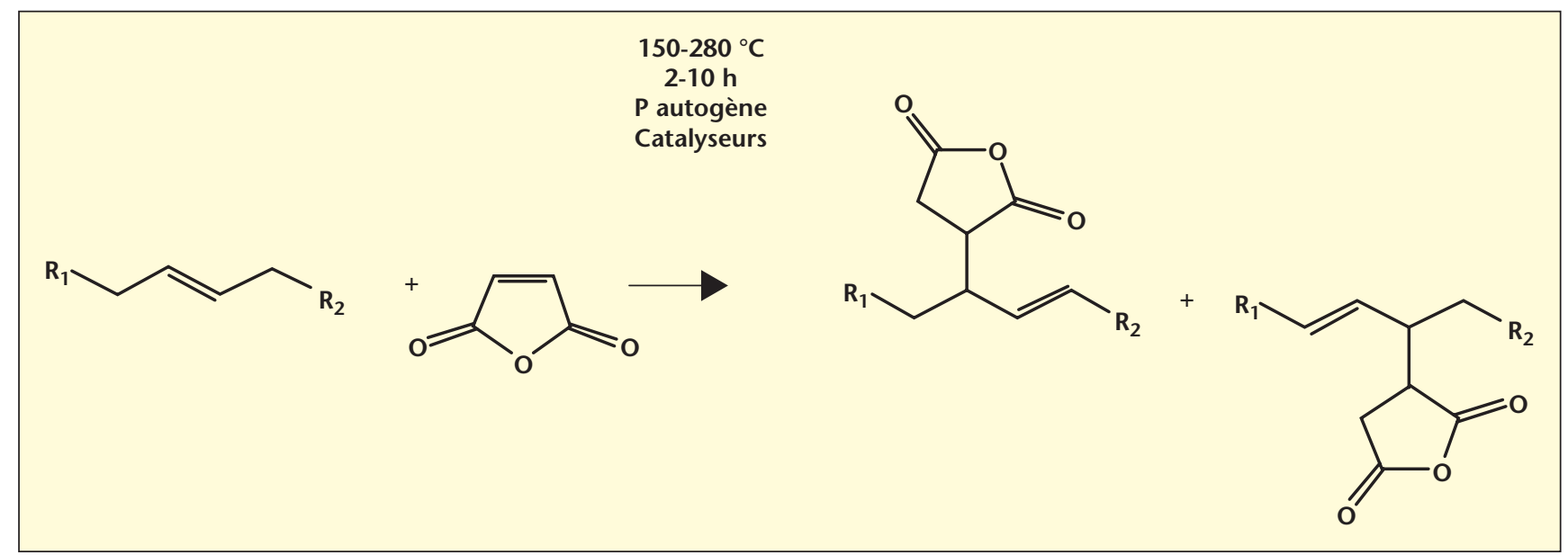

Figure 3. Les anhydrides d'alkényles succiniques lipidiques.

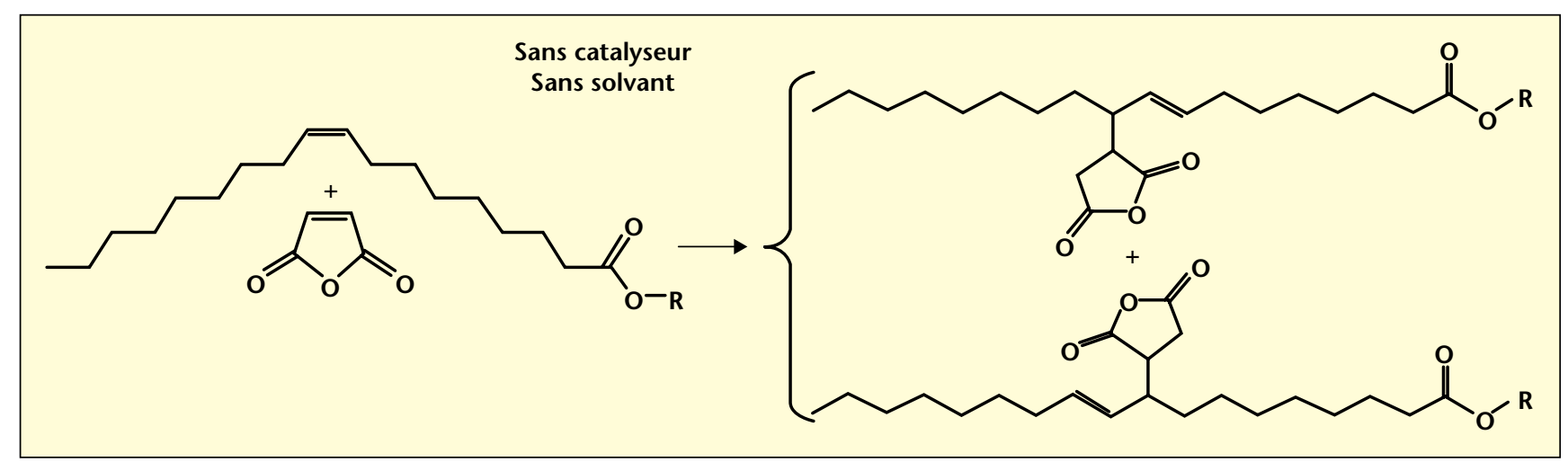

Figure 4. Les oléo-ASA obtenus par la ène-réaction entre des esters mono-insaturés d'huiles végétales et l'anhydride maléique (procédé n'utilisant ni solvant ni catalyseur). 


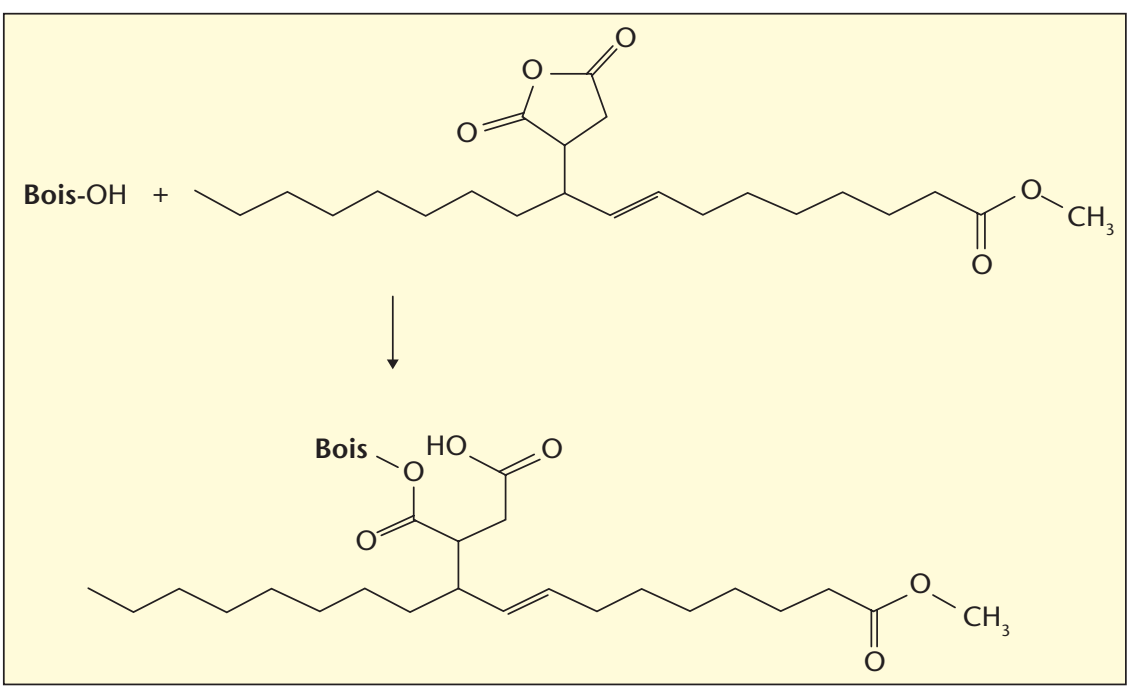

Figure 5. Réaction d'acylation d'abord sur la cellulose puis ensuite sur des substrats lignocellulosiques.

Les oléo-ASA obtenus par la ène-réaction entre des esters mono-insaturés d'huiles végétales et I'anhydride maléique a été réalisée et optimisée en mettant en œuvre un procédé propre n'utilisant ni solvant ni catalyseur [10].

Un mélange de deux régio-isomères constitue les oléo-ASA obtenus. Cette synthèse a été étudiée jusqu'à l'échelle pilote (200 litres).

Sur un plan fondamental, nous avons pu synthétiser une nouvelle famille de molécules d'origine végétale, non répertoriée dans les Chemical Abstracts [11].
L'un des objectifs scientifiques de ce travail a consisté à valider I'hypothèse selon laquelle un tel réactif amphiphile pourrait mieux pénétrer dans le réseau complexe lignocellulosique pour réagir ensuite avec les groupements fonctionnels hydrophiles.

Ces oléo-ASA ont donc été utilisés afin de rechercher les meilleures conditions de mise en œuvre de la réaction d'acylation d'abord sur la cellulose puis ensuite sur des substrats lignocellulosiques (figure 5).
Nous avons pu vérifier que l'augmentation du caractère polaire du réactif lipophile lié à cette fonctionnalisation, a permis d'une part d'atteindre l'objectif visé et d'autre part d'obtenir de nouveaux substrats « lignocellulosiqueslipidiques » propices à de nombreuses applications notamment dans deux domaines:

- la conservation des bois :

- les agents de collage dans l'industrie du papier d'écriture.

\section{La conservation des bois}

Au-delà de la validation de la réaction de greffages de chaînes lipidiques sur les fibres lignocellulosiques, les observations faites en microscopie électronique à balayage de diverses coupes de bois (pin sylvestre et hêtre) avant et après greffage montrent l'importance du remplissage des vaisseaux par l'oléo-ASA (figure 6). Les bois ainsi traités présentent un caractère hydrophobe mais montrent surtout l'efficacité de ce dérivé lipidique dans le cadre de la protection biologique du bois $[12,13]$. En effet, ces oléo-ASA ont permis d'atteindre la classe de risque biologique 3 incluant de surcroit l'action sur les termites sans variation dimensionnelle des pièces de bois. Ce résultat est important car il représente une alternative aux traitements actuels du bois d'œuvre qui utilisent des produits toxiques tels que l'arsenic, le chrome, les créosotes, etc. Suite à la directive "Biocides" 98/8/CE, ces produits nocifs pour l'homme et pour l'environnement seront interdits en

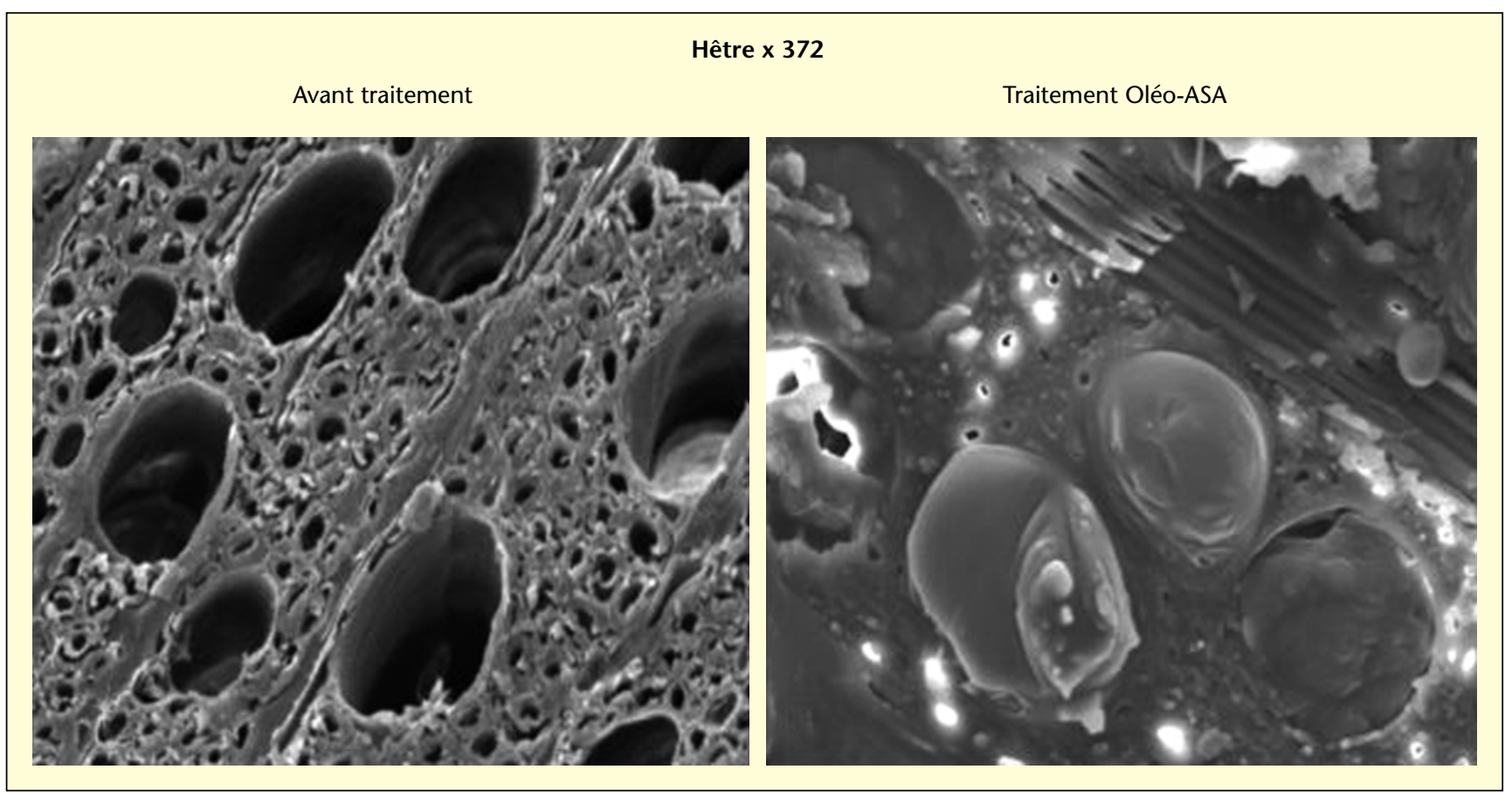

Figure 6. Après traitement, aucune variation dimensionnelle n'a été constatée. Grâce aux images obtenues par microscopie électronique à balayage, nous observons que l'ASAM est présent dans les vides cellulaires du bois. 
Europe à partir de 2008. À ces éléments il convient d'ajouter l'impact important qu'aura aussi dans ce secteur la réglementation Reach. Ces points renforcent tout l'intérêt d'utiliser des produits de substitution issus du carbone renouvelable dont l'innocuité sur l'homme et sur l'environnement sont avérés.

La modification chimique du bois avec des molécules lipidiques d'origine végétale permettant d'augmenter sa durabilité, marque une rupture technologique dans le domaine de la protection du bois d'œuvre [14]. L'extension à la classe de risque 4 des oléo-ASA fait actuellement l'objet d'un projet européen «SURFASAM ».

\section{Les agents de collage dans l'industrie du papier d'écriture}

En papeterie, un agent de collage permet de contrôler la pénétration de l'eau et des solutions aqueuses (encres) dans le papier d'écriture par la formation d'une surface hydrophobe sur la cellulose. II permet d'éviter l'effet dit « buvard».

Trois grandes familles d'agents de collage sont actuellement utilisées: les résines de colophane, les alkyles cétènes dimères (AKD) et les anhydrides d'alkényles succiniques (ASA). Ces derniers sont issus d'oléfines pétrochimiques préalablement isomérisées. Ils sont de plus en plus utilisés en Europe et aux USA.

Peut-on substituer, dans ce domaine d'application, les oléo-ASA aux ASA pétrochimiques?

À partir des oléo-ASA synthétisés, plusieurs tests de collage et diverses études ont été réalisés dans l'objectif d'établir une comparaison objective des performances entre ces agromolécules et les molécules d'ASA d'origine pétrochimique.

La mesure du caractère hydrophobe des papiers encollés est obtenue par le test normalisé $\mathrm{Cobb}_{60}$. Le $\mathrm{Cobb}_{60}$ de quelques molécules synthétisées à partir d'oléates d'alkyles linéaires montrent des capacités de collage équivalentes à celles de I'ASA pétrochimique.

L'étude de la cinétique d'hydrolyse de l'oléoASA en diacide suivie par infrarouge à transformées de Fourrier (FTIR) a permis de conclure que les molécules d'origine végétale s'hydrolysent deux fois plus lentement que leurs homologues pétrochimiques. Ce phénomène peut, en partie, être lié à la position centrale de la fonction anhydride dans la chaîne grasse. Cette fonction est alors protégée par les chaînes aliphatiques alors qu'elle a une position terminale plus accessible dans le cas des ASA pétrochimiques. De plus, cette cinétique d'hydrolyse plus lente des oléo-ASA permet d'expliquer que leurs émulsions sont plus stables que celles des ASA pétrochimiques. L'étude de l'entrainement à la vapeur d'eau des ASA fait ressortir que les oléo-
ASA présentent une résistance au phénomène de stripping dix fois plus importante.

L'ensemble de ces résultats constituent des arguments supplémentaires justifiant que les oléo-ASA ont une meilleure réactivité vis-à-vis de la cellulose que les ASA pétrochimiques. En effet, bien que les deux composés puissent former des liaisons esters au niveau de la fonction anhydride avec les hydroxyles de la cellulose, seul I'oléo-ASA peut, lui, être lié par des interactions supplémentaires qui mettent à contribution la fonction ester terminale.

La plus faible valeur de la cinétique d'hydrolyse et la faible aptitude au «stripping » des oléoASA constituent deux points importants qui sont favorables à l'utilisation des oléo-ASA en papeterie.

En effet, les ASA pétrochimiques en s'hydrolysant rapidement sont alors moins réactifs envers la cellulose. Ils ont en outre une forte tendance à être entrainés par l'eau et donc à encrasser les machines ainsi que les conduites d'aération en se redéposant.

L'utilisation d'oléo-ASA permettrait de diminuer le coût de nettoyage des machines puisque l'encrassement généré serait 10 fois inférieur à celui créé par la mise en œuvre des ASA pétrochimiques. Enfin, l'hydrolyse des oléoASA étant deux fois plus lente, leur utilisation serait synonyme d'une augmentation de la flexibilité du mode opératoire de collage.

Des essais de collage ont été réalisés avec succès à l'échelle pilote (100 kilos de papier par heure). Les essais à l'échelle industrielle (15 tonnes de papier par heure) seront réalisés en 2006.

D'un point de vue industriel et technologique pour la papeterie, ces travaux ont permis de mettre à jour de nouvelles molécules synthétisées à partir de ressources renouvelables, dont les qualités en tant qu'agents de collage sont supérieures à celles des dérivés pétrochimiques actuellement utilisés.

\section{Conclusion}

Ces résultats tentent modestement d'apporter leur participation au développement d'une lipochimie qui en s'associant à un autre constituant de la matière végétale (la lignocellulose et les lignocelluloses) contribue à améliorer I'ouverture économique des huiles végétales à travers l'exploitation d'oléo-produits. Les nouvelles technologies et les dérivés lipochimiques adaptés permettront d'atteindre une réactivité correcte entre les deux partenaires et par voie de conséquence de développer ce secteur d'application. En effet, chacun sait que la lipochimie, au-delà du biodiesel, a une place remarquable à prendre dans des domaines aussi variés que les biolubrifiants, les biosol- vants, les biodétergents, les bioadhésifs, les biovernis, les agromatériaux, la cosmétologie, etc.

Ce concept de lipochimie qui s'intègre parfaitement dans la notion de développement durable va contribuer au redéploiement d'une nouvelle chimie: la chimie verte du carbone renouvelable. II est certain qu'il existe là un gisement d'activités économiques propices à la création d'une agro-industrie très diversifiée et très prometteuse. En effet, les experts s'accordent pour dire que dans un futur très proche la chimie fine et la chimie de spécialité progresseront de façon très importante en s'appuyant surtout sur le carbone renouvelable au détriment du carbone fossile.

$L^{\prime}$ un des objectif du $7^{e}$ PCRD « Life sciences and biotechnology for sustainable non-food products and process » et également " The European Technology Platform for SUSTAINABLE CHEMISTRY - Suschemistry - Suschem nonfood chemistry » nous incitent à mobiliser nos forces dans ce sens. Les écoprocédés et les bioproduits issus de la lipochimie y occuperont évidemment une place non négligeable.

Un tel affichage confirme bien que nous sommes entrés dans l'ère du renouvelable et qu'une très belle aventure nous attend. II faut toutefois être très actifs car ce que l'on ne décidera pas aujourd'hui aura de grosses répercussions demain.

Remerciements. Nos remerciements vont d'abord aux industriels, aux institutionnels, aux interprofessions, aux experts technologiques et aux scientifiques qui nous ont soutenus et aidés dans la réalisation de nos projets. Sans les acteurs, administratifs, techniques et scientifiques $d u$ laboratoire de chimie agro-industrielle UMR 1010 Inra/INP - Ensiacet, ces résultats n'auraient jamais été obtenus. Chacun d'eux a apporté une contribution efficace au bénéfice de la lipochimie et de notre UMR. Nous tenons à les remercier très chaleureusement pour leur généreuse implication et pour leur constance.

\section{RÉFÉRENCES}

1. THIEBAUD S, BORREDON ME. Solvent-free wood esterification with fatty acid chlorides. Bioresour Technol $1995 ; 52$ : 169-73.

2. THIEBAUD S, BORREDON ME, BAZIARD G, SENOCQ F. Properties of wood esterified by fatty-acid chlorides. Bioresour Technol 1997 ; $59: 103-7$

3. THIEBAUD S, BORREDON ME. Analysis of the liquid fraction after esterification of sawdust with octanoyl chloride - Production of esterified hemicelluloses. Bioresour Technol 1998; $63: 139-45$. 
4. VACA-GARCIA C, THIEBAUD S, BORREDON ME, GOZZELINO G. Cellulose esterification with fatty acids and acetic anhydride in lithium chloride/N,N-dimethylacetamide medium. I Am Oil Chem Soc 1998 ; 75 : 315-9.

5. VACA-GARCIA C, BORREDON ME. Solvent free fatty acylation of cellulose and lignocellulosic wastes. Part 2 : reactions with fatty acids. Bioresour Technol 1999 ; 70 : 135-42.

6. VACA-GARCIA C, BORREDON ME, GASET A. Determination of the degree of substitution of mixed cellulose esters by elemental analysis. Cellulose $2001 ; 8$ : 225-31.

7. ORLIAC O, SILVESTRE F. Microwave esterification of sunflowers proteins in solvent-free conditions. Bioressource Technology 2003 ; 87 : 63-8.
8. VACA-GARCIA C, GIRARDEAU S, NICOLAS D, CARUEL H, BORREDON ME, GASET A. (1999, 2000), Procédé pour conférer un caractère hydrophobe à une matière solide cellulosique ou amylacée, Brevet FR 2789995523 puis étendu en PCT WO 00/50492.

9. MAGNE M, EL KASMI S, DUPIRE M, et al. Procédé de traitement de matières lignocellulosiques, notamment du bois ainsi qu'un matériau obtenu par ce procédé, Brevet FR 2838369. Publication demande internationale: WO 03/084723. 2003

10. QUESADA J, MORARD M, VACA-GARCIA C, BORREDON ME. Preparation of alkenyl succinic anhydrides from vegetable oil FAME. / Am Oil Chem Soc 2003 ; 80 : 281-6.

11. CANDY L, VACA-GARCIA C, BORREDON ME. Synthesis of alkenyl succinic anhydrides from methyl esters of high oleic sunflower oil. Eur J Lipid Sci Technol 2005 ; 107 : 3-11.
12. MORARD M, VACA-GARCIA C, STEVENS M, VAN ACKER J, BORREDON M. Technological properties of wood treated with methyl alkenoate succinic anhydrides (M-ASA) of vegetable origin. Durability increase, dimensional stability and mechanical resistance. Wood Sci Technol 2005 ; (Submitted for publication).

13. MORARD M, PIGNOLET O, VACA-GARCIA C, STEVENS M, VAN ACKER J, BORREDONM. Biological properties of M-ASA. Materials und Organismen 2005 ; (Submitted for publication).

14. MORARD M, VACA-GARCIA C, BORREDON ME, GASET A, LEMOR A, MAISONABE P. Procédé de traitement d'un matériau lignocellulosique, en particulier de bois, en vue de sa préservation et d'une amélioration de ses propriétés mécaniques, et matériau obtenu, Brevet FR 2833202. Publication demande internationale WO 03/049913. 2003. 